\title{
As leis de Newton e a estrutura Espaço-temporal da Mecânica Clássica
}

\author{
On Newton's Laws and the Space-time structure of Classical Mechanics \\ Camila A. Antunes ${ }^{1}$, Vinícius B. Galhardi ${ }^{1}$, Carlos A. Hernaski ${ }^{*}[0$ \\ ${ }^{1}$ Universidade Estadual de Londrina, Departamento de Física, Caixa Postal 10011, 86057-970, Londrina, PR, Brasil
}

Recebido em 06 de Janeiro, 2018. Revisado em 04 de Fevereiro, 2018. Aceito em 13 de Fevereiro, 2018.

\begin{abstract}
Neste artigo, discutimos a estrutura das leis de movimento da mecânica clássica advinda de hipóteses gerais de simetria do espaço-tempo e do entendimento dessa teoria como sendo uma descrição efetiva para fenômenos macroscópicos. Usando argumentos de análise dimensional inspirados nas técnicas de teoria de campos efetivas, sustentamos em favor da naturalidade das leis de Newton da mecânica clássica.
\end{abstract}

Palavras-chave: Simetrias, Mecânica Clássica, Leis de Newton, Teorias efetivas, Simetrias emergentes

In this article, we discuss the structure of the laws of motion of classical mechanics derived from general hypotheses of space-time symmetries and the understanding of this theory as an effective description for macroscopic phenomena. Using dimensional analysis arguments inspired in effective field theory techniques, we sustain in favor of naturalness of the Newton's laws of classical mechanics.

Keywords: Symmetries, Newton's Laws, Effective theories, Emergent symmetries

\section{Introdução}

De modo geral, a mecânica é uma área da Física que se propõe a descrever os princípios básicos que regem os movimentos dos corpos no espaço enquanto o tempo flui. Dessa forma, para sua completa consistência, deve-se definir precisamente o que se entende por tempo, espaço e objeto. Tais conceitos, evidentemente, extrapolam o escopo da mecânica e influenciam na descrição das demais áreas da Física. Além disso, diferentes noções dessas estruturas levam a distintas realizações da mecânica. O foco deste artigo é a análise da estrutura do que entendemos por mecânica clássica, que também nos referimos por mecânica Newtoniana.

De um ponto de vista moderno, a mecânica Newtoniana pode ser entendida como um limite de baixas velocidades (comparadas com a velocidade da luz) e de grandes distâncias (comparadas com o comprimento de onda de de Broglie) de uma mecânica quântica e relativística. Contudo, como sabemos, a concepção da mecânica Newtoniana se deu de forma completamente independente de suas generalizações e constitui, portanto, uma estrutura auto-consistente segundo suas premissas e hipóteses. Não queremos com isso dizer que ela é consistente com as observações em todas as escalas de distância e para quaisquer velocidades, mas sim que possui uma estrutura lógica consistente. As generalizações da mecânica Newtoniana servem, nesse sentido, para delimitar seu intervalo de validade experimental. Observado este intervalo de

*Endereço de correspondência: hernaski@uel.br validade, a mecânica Newtoniana se mostrou uma teoria de enorme sucesso fenomenológico.

Como geralmente ocorre no processo de construção de uma teoria científica, o desenvolvimento da mecânica Newtoniana traçou um caminho altamente sinuoso. Por tentar extrair princípios gerais de fenômenos facilmente acessíveis, diversos conceitos informais do cotidiano foram sendo incorporados na descrição científica, muitas vezes sem a devida precisão. A sistemática do método científico moderno desenvolvido por Galileu [1,2, constituiu um avanço importante na formulação da mecânica. Seguindo seus preceitos, Newton foi capaz de enunciar suas três leis do movimento de maneira muito próxima da forma que conhecemos atualmente 3 .

Neste artigo pretendemos analisar o papel que as simetrias do espaço-tempo da mecânica clássica podem desempenhar na própria formulação das leis do movimento.

A Física moderna se apropriou de maneira bastante eficiente do conceito de simetria em um sistema físico e de como os padrões simetria podem restringir a própria dinâmica do sistema. O formalismo Lagrangeano é particularmente útil para esse propósito, pois a função Lagrangeana que contém toda informação dinâmica do sistema, por ser uma grandeza escalar, deve ser invariante frente às transformações de simetria. Além disso, e também por isso, o formalismo Lagrangeano é singularmente adequado na conexão das simetrias do sistema com grandezas que se conservam durante sua evolução dinâmica (constantes de movimento), que se torna explícita 
no celebrado Teorema de Noether [4 7]. Essa conexão pode também ser discutida mesmo dentro do formalismo Newtoniano da mecânica clássica, embora de forma não tão sistemática [8]. A análise das restrições que as simetrias espaço-temporais impõem às leis de movimento nesse formalismo também já foi explorada 9]. Nesse caso, a invariância das leis físicas frente ao grupo de Galileu desempenha um papel crucial.

É o objetivo do presente artigo contribuir para essa discussão tratando como hipóteses básicas a invariância das leis físicas frente ao grupo de rotações e translações espaciais, translações temporais, além das simetrias discretas de reversão temporal e paridade. Entendemos que estas simetrias são de certa forma naturais pela economia de hipóteses necessárias, ao passo que a invariância sob boosts Galileanos precisa ser justificada de forma independente e procuramos relacioná-la com a hipótese da preditibilidade da mecânica clássica e da interpretação desta teoria como uma teoria efetiva para descrição dos fenômenos macroscópicos. Com estas hipóteses, demonstramos a validade das leis de Newton para um sistema de partículas clássicas fundamentais, que definiremos apropriadamente. De fato, para um tal sistema de partículas, demonstramos a validade de uma versão mais forte da terceira lei, onde a interação é mediada por uma força conservativa. A emergência macroscópica da versão usual da terceira lei e de forças não-conservativas é, então, discutida e demonstrada.

Este trabalho está organizado da seguinte maneira. Na Sec. 2 faremos uma breve revisão do conteúdo das três leis de Newton do movimento e definiremos quantidades de interesse para as discussões deste artigo. Em seguida, na Sec. 4 discutimos as simetrias espaço-temporais da mecânica clássica e suas implicações na forma das equações que regem a dinâmica dos objetos. Esta discussão é complementada com a interpretação da mecânica como uma teoria efetiva que descreve fenômenos numa escala de baixas energias comparada com a escala característica de uma teoria quântico-relativística. Nessa seção também analisamos o surgimento das forças não-conservativas na dinâmica macroscópica efetiva. Por fim, expomos nossas considerações finais.

\section{As leis de Newton do movimento}

Como resultado de séculos de observações e conhecimento acumulado, Newton foi capaz de condensar os princípios que regem a dinâmica dos corpos em suas três leis do movimento [10]. Numa formulação moderna, estas leis podem ser enunciadas da seguinte forma:

Primeira Lei Todo corpo persiste em seu estado de repouso ou de movimento retilíneo uniforme a menos que seja compelido a modificar este estado pela ação de forças sobre ele.
Segunda Lei A soma das forças que atuam sobre um corpo é igual ao produto de seu coeficiente de inércia pela sua aceleração:

$$
\vec{F}=m \vec{a} .
$$

Terceira Lei A toda ação que um corpo exerce sobre um segundo corpo, corresponde uma reação do segundo sobre o primeiro de mesma intensidade e sentido oposto.

A primeira lei, também chamada lei da inércia, serve para limitar o escopo da segunda lei, de modo que a validade desta última é restrita à classe de referenciais inerciais (aqueles que obedecem a lei da inércia). A segunda lei introduz o coeficiente de inércia $m$, que é uma constante positiva característica de cada objeto. A lei de forças $\vec{F}$ deve ser obtida, em última análise, de forma empírica e a solução do problema mecânico pressupõe seu conhecimento. Além disso, a segunda lei afirma que o estado de um objeto é completamente determinado por sua posição e velocidade em qualquer instante do movimento, pois qualquer derivada da posição de ordem maior ou igual a dois pode ser obtida recorrentemente de (1). De certo modo, a terceira lei complementa a primeira, pois um objeto composto, onde os seus constituintes interagem somente entre si e não sofrem ação externa, obedecerá a lei da inércia. Ambas as leis equivalem à conservação do momento linear total do sistema.

Como bem sabemos, desde sua concepção, esse conjunto de leis tem sido usado com sucesso para descrição de vários sistemas físicos. Um de seus grandes triunfos apareceu já no terceiro volume dos Principia [3], onde Newton demonstrou que essas leis, combinadas com sua lei da Gravitação universal, eram capazes de descrever as leis de Kepler dos movimentos planetários.

Antes de seguirmos adiante com nossa revisão, esclarecemos em que escala consideramos o regime de validade das leis de Newton. Como é conhecido, tais leis são capazes de descrever os fenômenos observados numa escala macroscópica e de baixas velocidades. Como já mencionamos na introdução deste artigo, a relatividade especial introduz uma nova constante com dimensão de velocidade que coincide com a velocidade da luz no vácuo $c$ e que pode ser usada como referência para separar movimentos lentos, $v \ll c$, de movimentos rápidos, $v \sim c$. O conceito de macroscópico, por outro lado, pode variar de sistema para sistema. O importante é que os efeitos quânticos não sejam importantes o suficiente para invalidar o tratamento clássico. Em geral, podemos definir uma escala de comprimento que separa os fenômenos quânticos dos fenômenos clássicos, como o comprimento de onda de de Broglie $\lambda=h / p$, onde $h \approx 6,6 \times 10^{-34}$ J.s é a constante de Planck e $p=m v$ é o módulo do momento linear de uma objeto de massa $m$ e velocidade $\vec{v}$. Assim, se a escala de comprimento característica do sistema $d$ é tal que $d \gg \lambda$, podemos tratar classicamente os fenômenos associados. 
Na maioria das aplicações do formalismo clássico, não precisamos nos preocupar com a verificação destes limites, pois grande parte dos sistemas analisados são objetos do cotidiano e satisfazem de maneira óbvia uma dinâmica clássica. Contudo, mesmo nesta situação, é preciso fazermos algumas qualificações. Como referência de objeto macroscópico deste tipo, consideramos um corpo composto por um número de moléculas da ordem de $1 \mathrm{~mol} \approx 6,022 \times 10^{23}$. Se formos considerando porções cada vez menores deste objeto, eventualmente chegaremos no menor constituinte com comportamento ainda clássico, ou seja, que podemos atribuir um estado prescrevendo simultaneamente a posição e velocidade do mesmo e aplicar as leis de Newton para descrever sua dinâmica. É provável que esse constituinte, que definiremos como partícula fundamental clássica, compreenda algumas poucas moléculas e suas dimensões podem ser desprezadas frente às dimensões do objeto macroscópico.

Apesar de uma partícula fundamental clássica possuir estrutura interna, podemos, em geral, realizar experimentos onde estes graus de liberdade internos não são excitados. Dessa forma, a dinâmica total do sistema pode ser descrita de forma inteiramente clássica. Contudo, o grande número de partículas fundamentais torna impraticável a solução do problema mecânico. Além disso, uma tal solução, ainda que possível, poderia não ser de grande utilidade devido à grande quantidade e complexidade da informação fornecida. Em outras palavras, quando pretendemos analisar o problema mecânico de um bloco deslizando num plano inclinado, não estamos realmente interessados na possível dinâmica dos graus de liberdade microscópicos do bloco, mas sim na dinâmica dos poucos graus de liberdade que emergem macroscopicamente e descrevem o movimento do bloco como um todo.

Pode também acontecer de que além dos graus de liberdade macroscópicos, também estejamos interessados na manifestação macroscópica da dinâmica dos graus de liberdade internos. Isto ocorre quando a realização de trabalho sobre um sistema não excita somente os graus de liberdade macroscópicos, mas também a miríade de graus de liberdade internos. Neste caso, é comum parametrizarmos essa dinâmica em termos de interações macroscópicas efetivas que, como veremos, dão origem às forças não-conservativas.

\section{Simetrias e Leis de Conservação}

Apesar de as propriedades de simetria do espaço-tempo não terem sido explicitamente invocadas na construção das leis de Newton, pode-se averiguar a posteriori quais são essas propriedades e suas consequências na dinâmica dos sistemas físicos. Notemos, por exemplo, que o lado direito da segunda lei de Newton (1) não introduz nenhuma estrutura externa referente ao espaço-tempo. Ou seja, não há nenhum ponto ou direção espacial ou temporal privilegiadas advindas desse termo. Assim, as propriedades de simetria de um sistema com $N$ partículas, não necessariamente fundamentais, dependem da forma dos campos de forças

$$
\vec{F}_{i}\left(\vec{x}_{1}, \ldots, \vec{x}_{N} ; \dot{\vec{x}}_{1}, \ldots, \dot{\vec{x}}_{N} ; t\right), \quad \text { com } i=1, \ldots N
$$

onde $\vec{x}_{j}$ e $\dot{\vec{x}}_{j}$ correspondem ao vetor posição e velocidade da $j$-ésima partícula, respectivamente e $\vec{F}_{i}$ é a força sobre a $i$-ésima partícula devida as demais partículas de um sistema isolado.

Como já mencionamos na seção anterior, e veremos explicitamente adiante, as propriedades de simetrias espaçotemporais nos permitem atribuir uma função escalar $U\left(\vec{x}_{1}, \ldots, \vec{x}_{N_{f}}\right)$ a um sistema com $N_{f}$ partículas fundamentais clássicas, de tal modo que a força (2) sobre tais partículas seja dada por $\vec{F}_{a}=-\vec{\nabla}_{a} U$, com $a=1, \ldots N_{f}$. De maneira mais geral, se a dinâmica dos graus de liberdade internos puder ser completamente ignorada, podemos descrever a dinâmica do sistema macroscópico em termos da função energia potencial macroscópica $U^{M}\left(\vec{x}_{1}, \ldots, \vec{x}_{N_{c}}\right)$, com a força sobre a $j$-ésima partícula composta sendo dada por $\vec{F}_{j}=-\vec{\nabla}_{j} U^{M}$, com $j=1, \ldots N_{c}$. Neste caso, os argumentos da função $U^{M}$ devem ser interpretados como coordenadas macroscópicas. Um exemplo são coordenadas do centro de massa das partículas compostas. Tipicamente pode-se supor $N_{c} \ll N_{f}$, pois cada partícula composta do sistema contém em geral uma grande quantidade de partículas fundamentais clássicas. Sistemas macroscópicos, cujas interações são descritas por uma função $U^{M}$, são ditos conservativos. Para esses sistemas, a segunda lei de Newton se escreve

$$
-\vec{\nabla}_{i} U^{M}=m_{i} \vec{a}_{i} .
$$

As simetrias espaço-temporais podem ser analisadas em termos das simetrias da função energia potencial $U(\vec{x}, t)$ e, como mostraremos, estão associadas a grandezas conservada: 1 Esta discussão é bastante conhecida na literatura [8], mas por completeza, repetiremos algumas considerações neste trabalho.

Consideremos, por exemplo, que a dinâmica do sistema isolado seja dado num espaço homogêneo. Neste caso, uma translação rígida infinitesimal do sistema como um todo,

$$
\vec{x}_{i}^{\prime}=\vec{x}_{i}+\vec{\epsilon}, \quad i=1, \ldots, N
$$

deixa $U\left(\vec{x}_{i}, t\right)$ invariante. Assim,

$$
d U=\sum_{i=1}^{N} \vec{\nabla}_{i} U \cdot \vec{\epsilon}=-\sum_{i=1}^{N} \vec{F}_{i} \cdot \vec{\epsilon}=0 .
$$

Sendo $\vec{\epsilon}$ um vetor infinitesimal arbitrário e usando (1), temos

$$
\frac{d}{d t} \sum_{i=1}^{N} \vec{p}_{i}=0
$$

${ }^{1}$ Neste ponto, é indiferente se discutimos essa conexão em termos da função microscópica $U$ ou macroscópica $U^{M}$, com a ressalva de que, como mencionado acima, deve-se interpretar as coordenadas $\vec{x}$ de $U^{M}$ como coordenadas do centro de massa das partículas compostas. 
com $\vec{p}_{i}=m_{i} \vec{v}_{i}$ sendo o momento linear da $i$-ésima partícula. Portanto, a simetria por translações (4) leva à conservação do momento linear total do sistema.

A simetria por rotações rígidas do sistema completo é uma consequência da isotropia espacial. Neste caso, uma rotação infinitesimal por um ângulo $\delta \theta$ em torno de um eixo representado por um vetor unitário $\hat{n}$, afeta os vetores posição das partículas da seguinte forma:

$$
\vec{x}_{i}^{\prime}=\vec{x}_{i}+\delta \theta \hat{n} \times \vec{x}_{i}
$$

A invariância de $U^{M}$ sob estas transformações implica

$$
\begin{aligned}
d U & =\sum_{i=1}^{N} \vec{\nabla}_{i} U \cdot\left(\overrightarrow{\delta \theta} \times \vec{x}_{i}\right)=-\sum_{i=1}^{N} \vec{F}_{i} \cdot\left(\overrightarrow{\delta \theta} \times \vec{x}_{i}\right) \\
& =-\sum_{i=1}^{N} \frac{d \vec{p}_{i}}{d t} \cdot\left(\overrightarrow{\delta \theta} \times \vec{x}_{i}\right)=0
\end{aligned}
$$

com $\overrightarrow{\delta \theta} \equiv \delta \theta \hat{n}$ e fazendo uso de (1). Da ciclicidade do produto misto e do fato de que $\vec{p}_{i} \times \vec{v}_{i}=m_{i} \vec{v}_{i} \times \vec{v}_{i}=0$, podemos reescrever a última igualdade em (8) como

$$
\overrightarrow{\delta \theta} \cdot \frac{d}{d t}\left(\sum_{i=1}^{N} \vec{x}_{i} \times \vec{p}_{i}\right)=0 .
$$

Como $\overrightarrow{\delta \theta}$ é arbitrário, obtemos a conservação do momento angular total do sistema,

$$
\frac{d}{d t}\left(\sum_{i=1}^{N} \vec{L}_{i}\right)=0,
$$

$\operatorname{com} \vec{L}_{i} \equiv \vec{x}_{i} \times \vec{p}_{i}$

Por fim, consideramos a simetria por translações temporais:

$$
t^{\prime}=t+\lambda
$$

A invariância dinâmica do sistema por translações temporais implica que $U$ só deve depender de $t$ implicitamente por meio da evolução dinâmica de $\vec{x}(t)$. Ou seja,

$$
\frac{\partial U}{\partial t}=0
$$

Desta forma, a variação total de $U$ durante a evolução dinâmica do sistema é dada por

$$
\begin{aligned}
d U & =\sum_{i=1}^{N} \vec{\nabla}_{i} U \cdot \vec{v}_{i} d t=-\sum_{i=1}^{N} m_{i} \frac{d \vec{v}_{i}}{d t} \cdot \vec{v}_{i} d t \\
& =-d\left(\sum_{i=1}^{N} \frac{1}{2} m_{i} \vec{v}_{i}^{2}\right) .
\end{aligned}
$$

Definindo a energia cinética total por $T \equiv \sum_{i=1}^{N} \frac{1}{2} m_{i} \vec{v}_{i}^{2}$, temos então a conservação da energia total do sistema, $E=T+U$, pois de 13 , obtém-se

$$
d E=d(U+T)=0 \text {. }
$$

A investigação sistemática da relação entre simetrias contínuas do sistema físico e grandezas conservadas fica mais clara no formalismo Lagrangeno da mecânica. Este formalismo pode ser visto como uma concepção independente da mecânica clássica com seus próprios princípios. Postula-se a existência de uma função escalar $\mathcal{L}(\vec{x}, \vec{v}, t)$ que contém toda informação dinâmica do sistema. Dentre todas as trajetórias possíveis entre pontos iniciais e finais, $\vec{x}\left(t_{0}\right)$ e $\vec{x}\left(t_{f}\right)$, a que efetivamente se realiza fisicamente é aquela que extremiza o funcional ação $S[\vec{x}(t)]=\int_{\vec{x}_{0}}^{\vec{x}_{f}} d t \mathcal{L}(\vec{x}(t), \dot{x}(t), t)$. Este processo culmina nas equações de movimento no formalismo Lagrangeano:

$$
\frac{d}{d t}\left(\frac{\partial \mathcal{L}}{\partial \dot{\vec{x}}}\right)-\left(\frac{\partial \mathcal{L}}{\partial \vec{x}}\right)=0
$$

Não é nosso propósito fazer uma revisão detalhada do formalismo Lagrangeano. Aqui só queremos enfatizar que a equação fundamental da dinâmica Newtoniana (3) para sistemas conservativos pode ser vista como advinda de um processo de extremização funcional. De fato, considerando a função Lagrangeana $\mathcal{L}=T-U$, mostra-se que as equações (3) e (15) são, neste caso, equivalentes.

\section{A emergência das leis de Newton}

Esta seção compreende a parte principal deste trabalho. Para facilitar a argumentação, dividimos a discussão em quatro subseções. Nas três primeiras, construímos sistematicamente as leis de movimento para objetos idealizados constituintes dos corpos macroscópicos. Por fim, discutimos a emergência das leis de Newton para descrição dinâmica desses objetos macroscópicos.

\subsection{Vínculos devidos às simetrias espaço-temporais}

Diferentemente da seção anterior, onde seguimos o desenvolvimento histórico das Leis de Newton e as consequentes propriedades das simetrias do espaço-tempo, nesta seção colocaremos num patamar mais fundamental as propriedades de simetria espaço-temporais e investigaremos como essas restringem a forma das leis do movimento.

Estas leis devem descrever como os corpos se movem no espaço enquanto o tempo flui sob o efeito de suas interações mútuas. Conforme nossa discussão na Sec. 2 , supomos os objetos serem compostos por partículas fundamentais clássicas, que são os menores constituintes da matéria que obedecem uma dinâmica clássica. Esta idealização nos permite atribuir uma série de propriedades cinemáticas a esses objetos, como posição e suas derivadas temporais de ordem arbitrária, de modo tão preciso quanto necessitarmos. A interação entre essas partículas é assumida ser instantânea e local. A primeira propriedade permite aos observadores formar uma noção absoluta de instantaneidade que por sua vez permite adotar uma estrutura com espaço e tempo absolutos. Além 
disso, assumimos a existência de uma classe de observadores denominados inerciais que percebe as propriedades geométricas do espaço tri-dimensional como sendo homogêneo e isotrópicd ${ }^{2}$ o fluxo temporal homogêneo, além das invariâncias discretas de paridade e reversão temporal. Por local, consideramos que as interações decaem com a distância entre as partículas, permitindo-nos definir de maneira prática a noção de partícula livre como sendo uma partícula suficientemente afastadas das demais partículas do universo.

Para fazermos progresso no nosso objetivo de encontrar as leis de movimento a partir de princípios de simetria, devemos discutir como fixamos o estado de uma partícula, ou seja, o número de informações cinemáticas que devemos fornecer num dado instante para que o estado posterior seja dado univocamente. É importante enfatizarmos que nossa busca pelas equações de movimento só faz sentido se o número de informações contidas num dado estado for finito. Consideremos, por exemplo, uma trajetória no espaço parametrizada pelo tempo e descrita por uma função analítica. Esta pode ser escrita como uma série de Taylor em torno de um instante fixo arbitrário $t_{0}$, ou seja,

$$
\vec{x}(t)=\sum_{n=0}^{\infty} \frac{\vec{x}_{0}^{(n)}}{n !}\left(t-t_{0}\right)^{n},
$$

onde $\vec{x}_{0}^{(n)}$ denota $\left.\frac{d^{n} x(t)}{d t^{n}}\right|_{t=t_{0}}$. Assim, se pudermos fixar arbitrariamente toda a série infinita de condições iniciais $\vec{x}_{0}^{(n)}$, fixamos automaticamente a solução completa $\vec{x}(t) \mathrm{e}$ não necessitaríamos de uma equação de movimento para fornecer essa solução. Além disso, uma situação como esta forneceria na prática uma mecânica impreditível, uma vez que uma série infinita de medidas seria necessária para fixar o estado inicial. Assim, adotamos como princípio a preditibilidade da mecânica e postulamos que apenas um número finito $s>0$ de condições iniciais é necessário para fixar o estado de um partícula univocamente ou, de forma equivalente, que existe uma função empírica que relaciona a $s$-ésima derivada temporal de $\vec{x}(t)$ com as derivadas de ordem menor, isto 3

$$
0=\vec{\beta}\left(\vec{x}, \vec{x}^{(1)}, \ldots, \vec{x}^{(s)} ; t\right) .
$$

Antes de tentarmos restringir a forma da função $\vec{\beta}$ com argumentos de simetria, notamos que, sendo (17) uma equação diferencial de ordem $s$, sua solução deve depender de $s$ condições iniciais, como queríamos. Estas condições caracterizam univocamente o estado de movimento de uma partícula livre. Denotemos este estado inicial por $\mathcal{X}_{0}=\left[\vec{x}_{0}, \vec{x}_{0}^{(1)}, \vec{x}_{0}^{(2)}, \ldots, \vec{x}_{0}^{(s-1)}\right]$. Para construir um outro estado inicial arbitrário a partir de um dado $\mathcal{X}_{0}$, é necessário interagir com a partícula e esta interação deve fornecer a regra pela qual a igualdade (17)

${ }^{2}$ Podendo ser identificada como o espaço Euclidiano $E^{3}$.

${ }^{3} \mathrm{O}$ fato de $\vec{\beta}$ ser uma função vetorial expressa o fato de que devemos ter uma equação de vínculo para cada componente do vetor $\vec{x}^{(s)}$. é violada. Da mesma maneira, a interação mútua entre as partículas de um sistema faz com que cada partícula se mova de tal modo que $\vec{\beta}_{i} \neq 0$. Neste caso mais geral tem-se

$$
\vec{F}_{i}=\vec{\beta}_{i}
$$

com $\vec{F}_{i}$ representando a lei força que as demais partículas exercem sobre a $i$-ésima.

Em breve discutiremos os vínculos impostos pelos argumentos de simetria à função que descreve a interação $\vec{F}_{i}$. Por ora, só queremos enfatizar o papel que essa função desempenha na própria construção dos estados iniciais das partículas, pois a mera existência de um estado do tipo $\mathcal{X}_{0}$ viola as simetrias assumidas até aqui, como a homogeneidade - quebrada pela realização do ponto $\vec{x}_{0}$ - e isotropia - pelas direções dos $\vec{x}^{(n)}$ — por exemplo. O fato de a equação (17) exibir tais simetrias, implica que o sistema deve ser preparado por um agente externo ou, em outras palavras, deve interagir com outro sistema, e esta interação é então desligada para $t>t_{0}$. Isto induz uma quebra espontânea de simetria que realiza o estado inicial $\mathcal{X}_{0}$.

Voltemos nossa atenção às restrições que podemos impor ao lado direito de (18) levando em conta argumentos de simetria. A homogeneidade espaço-temporal exige que não haja dependência explícita em $\vec{x}$ e $t$, ao passo que a isotropia espacial exige que

$$
\vec{\beta}\left(R \vec{x}, \ldots, R \vec{x}^{(s)}\right)=R \vec{\beta}\left(\vec{x}, \ldots, \vec{x}^{(m)}\right),
$$

com $R$ sendo uma matriz de rotação. Nossa notação é tal que $R \vec{x}$ é um vetor com componentes $(R \vec{x})_{a}=$ $\sum_{b=1}^{3} R_{a b} x_{b}$. A atuação nos demais vetores se dá de forma análoga.

Enfatizamos que até este momento estamos falando de uma única partícula movendo-se num referencial inercial. Essa equação pode ser vista como definidora dessa classe de sistemas de referência, pois estabelece o movimento das partículas livres. Dessa maneira, $\vec{\beta}$ deve ser uma função linear dos $\vec{x}^{(n)}$ — termos não lineares caracterizam auto-interação - e $n$ deve ser um inteiro par para preservar a invariância por reversão temporal. Destas considerações, temos

$$
\vec{\beta}=\sum_{n=1}^{(m+1) / 2} \lambda_{2 n} \vec{x}^{(2 n)}, \text { com } m \text { ímpar, }
$$

e os $\lambda_{2 n}$ são constantes que, conforme discutiremos mais adiante, não podem ser completamente arbitrárias.

Para progredir, poderíamos proceder como é feito em outras abordagens, invocando o princípio da relatividade Galileana, que é motivado pela observação e assegura que um observador que se movimenta com velocidade constante em relação a outro observador inercial é também inercial. Outro fato, também experimental, é que o estado de uma partícula é univocamente descrito em termos de sua posição e velocidade num dado instante. Com estes requerimentos extras, seríamos capazes de restringir 
a forma de $(19)$ ao ponto de termos $\vec{a}=0$ para uma partícula livre, o que nos daria a lei da inércia da mecânica Newtoniana. Contudo, neste artigo queremos explorar ao máximo as restrições das leis de Newton advindas das hipóteses de homogeneidade e isotropia espaço-temporal e de condições gerais como preditibilidade e estabilidade. Se poderia argumentar que estas hipóteses se justificam, em última análise, também da observação. Porém, nós entendemos que essas são na verdade condições naturais dentro do cenário espaço-temporal da mecânica clássica e, portanto, a introdução de outras estruturas é que necessitaria de uma justificativa plausível.

Contudo, não podemos restringir ainda mais a forma da função (19) apenas com os argumentos de simetria discutidos até aqui e entendendo a abordagem Newtoniana da mecânica como uma teoria consistente e isolada de uma fenomenologia microscópica mais fundamental. Neste sentido, o formalismo canônico da mecânica oferece algumas vantagens. Analisemos, por exemplo, a questão do número máximo de derivadas temporais da posição na equação de movimento. O fato de as equações de movimento advirem de um princípio variacional implica numa equação diferencial de ordem par e permite dividir as variáveis dinâmicas em pares posição-momento generalizados. Mostra-se, então, que a função Hamiltoniana não é positivo-definida e, portanto, leva à uma evolução temporal instável 11].

\subsection{A mecânica como uma teoria efetiva}

Uma maneira de incorporar termos com derivadas de ordens superiores na função Lagrangeana é considerálos como perturbações dos termos de ordem mais baixa. Esta situação aparece naturalmente nas descrições em termos de uma Lagrangeana efetiva, que se propõe a descrever uma classe de fenômenos numa região específica de intervalos de tempo, distância, energia, etc. Este tipo de abordagem é inspirado na Teoria Quântica de Campos, onde se usa o fluxo do grupo de renormalização para incorporar efeitos de graus de liberdade associados a altas energias numa descrição efetiva de energia mais baixa do sistema contendo um número menor de graus de liberdade 12,13 .

A filosofia da abordagem sistemática das teorias effetivas em Teoria de Campos pode ser incorporada e adequada para os diversos ramos da Física. Neste sentido, como já enfatizamos anteriormente, a mecânica clássica pode ser entendida como uma teoria macroscópica emergente de um cenário microscópico envolvendo mais graus de liberdade.

Vejamos como essa visão efetiva da mecânica e análise dimensional [14] pode nos guiar para restringirmos a forma da equação 17). Como estamos interessados neste momento apenas na teoria livre, podemos nos concentrar somente numa escala de tempo $T_{0}$ característica de um dado sistema microscópico. Isto se deve ao fato de que em 20] os termos só diferem entre si pelo número de derivadas temporais atuando em $\vec{x}(t)$. A descrição física do sistema na escala microscópica $T_{0}$ é entendida como fornecedora de uma descrição efetiva para fenômenos que ocorrem em escalas $\Delta t \gg T_{0}$, e que suporemos serem descritos pela mecânica Newtoniana. A supressão de graus de liberdade característicos da escala microscópica que não podem ser resolvidos na escala $\Delta t$ é parametrizada em termos de parâmetros efetivos dos termos que aparecem com uma expansão da função $\vec{\beta}$ nas variáveis dinâmicas do sistema. Da mesma forma que ocorre nas teorias efetivas descritas acima, esperamos que os aspectos robustos da dinâmica do sistema sejam descritos por um conjunto finito de termos a menos de uma classe infinita que fornece correções da ordem de $\left(\frac{T_{0}}{\Delta t}\right)^{n}$ dos efeitos descritos pelo primeiro conjunto. De fato, consideramos a razão entre dois termos sucessivos gerais desta expansão $\left|\frac{\mathcal{O}^{2(n+1)}}{\mathcal{O}^{2 n}}\right|$. A análise dimensional da discussão anterior nos fornece

$$
\left|\frac{\mathcal{O}^{2(n+1)}}{\mathcal{O}^{2 n}}\right|=\left|\frac{\lambda_{2(n+1)} \vec{x}^{(2(n+1))}}{\lambda_{2 n} \vec{x}^{(2 n)}}\right| \sim \frac{g_{2(n+1)}}{g_{2 n}}\left(\frac{T_{0}}{\Delta t}\right)^{2},
$$

onde os $g_{2 n}$ 's são constantes adimensionais que pelo critério de naturalidade devem ser da ordem de 1 . Vemos assim que os sucessivos termos da série 20) são suprimidos pelo fator $\left(\frac{T_{0}}{\Delta t}\right)^{2}$. Para estimarmos um valor aproximado para este fator, consideramos, por exemplo, a escala de tempo onde fenômenos quânticos e relativísticos passam a ser importantes. Tomemos a escala atômica, que é da ordem de $10^{-10} \mathrm{~m}$, e a velocidade da luz $\sim 10^{8} \mathrm{~m} / \mathrm{s}$, e obtemos uma escala de tempo da ordem de $T_{0} \sim 10^{-18} s$. Para intervalos da ordem de milissegundos, esse fator fornece correções da ordem de $10^{-30}$, que podem ser seguramente desprezados na escala das medidas macroscópicas da mecânica clássica.

Desse modo, podemos reter apenas o primeiro termo da expansão 20, e a equação 17 nos dá a lei da inércia como conhecemos

$$
\vec{a}=0
$$

Assim, recuperamos o resultado de que o estado de uma partícula é caracterizado somente por sua posição e velocidade. Além disso, obtemos o grupo de Galileu como o grupo de simetrias mais geral das leis da mecânica clássica.

\subsection{Propriedades da interação}

Tendo obtido a lei de movimento para partículas livres, retornemos nossa atenção ao lado esquerdo da segunda lei (18), ou seja, às propriedades da interação entre as partículas.

A função $\vec{F}_{i}$ é o que chamamos de força sobre a partícula $i$. A forma específica dessas funções deve ser obtida experimentalmente e é o que define o sistema de partículas. O fato de a interação ser instantânea- para uma dado instante $t$, as partículas só devem ter conhecimento 
das posições das demais partículas - permite-nos escrever $\vec{F}_{i}$ apenas como função das coordenadas dessas partículas. Além disso, a homogeneidade temporal impede uma dependência explícita no tempo. Da homogeneidade espacial, $\vec{F}_{i}$ deve se manter invariante se fizermos uma translação rígida do sistema como um todo. Este fato implica em $\vec{F}_{i}$ ser função apenas das diferenças $\vec{x}_{j}-\vec{x}_{k}$ entre as coordenadas. Por ser uma grandeza vetorial, as forças que agem sobre uma partícula se adicionam como tal. Podemos inferir, então, que a força resultante $\vec{F}_{i}$ é o resultado da soma das forças $\vec{F}_{i j}$ que a $j$-ésima partícula exerce sobre a $i$-ésima, com $j \neq i$, ou seja,

$$
\vec{F}_{i}=\sum_{j \neq i} \vec{F}_{i j}\left(\vec{x}_{i}-\vec{x}_{j}\right)
$$

onde novamente usamos as propriedades de simetria para expressar que a força entre as partículas $i$ e $j$ só dependem do vetor posição relativo entre as mesmas. Da mesma maneira que impomos a condição de invariância 19 na função $\vec{\beta}$ proveniente da isotropia espacial, temos que

$$
\vec{F}_{i j}\left(R \vec{x}_{i j}\right)=R \vec{F}_{i j}\left(\vec{x}_{i j}\right)
$$

com $\vec{x}_{i j} \equiv \vec{x}_{i}-\vec{x}_{j}$. Esta condição nos permite obter

$$
\vec{F}_{i j}\left(\vec{x}_{i j}\right)=F_{i j}\left(x_{i j}\right) \hat{x}_{i j},
$$

onde as quantidades escritas sem o símbolo de vetor significam o módulo da quantidade vetorial correspondente e $\hat{x}_{i j}$ é o vetor unitário na direção de $\vec{x}_{i j}$.

A equação 25) já nos assegura a validade da terceira lei de Newton para interação entre estas partículas fundamentais, pois $\vec{x}_{i j}=-\vec{x}_{j i}$ e $F_{i j}\left(x_{i j}\right)=F_{i j}\left(x_{j i}\right)=$ $F_{j i}\left(x_{i j}\right)^{4}$ Além disso, podemos definir a função escalar

$$
\begin{aligned}
U_{i j}\left(x_{i j}\right) & =-\int_{\left(\vec{x}_{i j}\right)_{0}}^{\vec{x}_{i j}} \vec{F}_{i j}\left(\vec{x}_{i j}^{\prime}\right) \cdot d \vec{x}_{i j}^{\prime} \\
& =-\int_{\left(x_{i j}\right)_{0}}^{x_{i j}} F_{i j}\left(x_{i j}^{\prime}\right) d x_{i j}^{\prime}
\end{aligned}
$$

com $\left(\vec{x}_{i j}\right)_{0}$ sendo um vetor arbitrário de referência e, por conveniência, supomos que $U\left(\left(x_{i j}\right)_{0}\right)=0$, de modo que

$$
\vec{F}_{i j}=-\vec{\nabla}_{i} U_{i j}=\vec{\nabla}_{j} U_{i j}=-\vec{F}_{j i}
$$

Esta definição de uma energia potencial $U_{i j}$ para o par de partículas $i j$, juntamente com expressão 23 para $\vec{F}_{i}$, nos permite definir a energia potencial de interação da partícula $i$ com o restante do sistema por $U_{i}=\sum_{j \neq i} U_{i j}$, pois $\vec{F}_{i}=-\sum_{j \neq i} \vec{\nabla}_{i} U_{i j}=-\vec{\nabla}_{i} \sum_{j \neq i} U_{i j}=-\vec{\nabla}_{i} U_{i}$. Podemos ir além e incluir nesta última expressão todos as demais funções $U_{j}$ com $j \neq i$, pois o gradiente $\vec{\nabla}_{i}$ atua trivialmente nos termos $U_{j k}$ que formam $U_{j}$, com

\footnotetext{
${ }^{4} F_{i j}$ depende de $\vec{x}_{i j}$ apenas por seu módulo $x_{i j}$ e, portanto, a notação $F_{i j}\left(x_{i j}\right)$ é de certa forma redundante. Seria suficiente expressarmos $F_{i j}$ por $F\left(x_{i j}\right)$, o que nos leva de maneira óbvia à condição $F_{i j}=F_{j i}$.
}

exceção do termo com $k=i$, que já está incluído em um dos termos de $U_{i}$. Assim, podemos levar em conta essa duplicidade e definir a função energia potencial do sistema completo por $U=\sum_{i<j} U_{i j}=\frac{1}{2} \sum_{i \neq j} U_{i j}$, de modo que

$$
\vec{F}_{i}=-\vec{\nabla}_{i} U
$$

A segunda lei de Newton aplicada às partículas fundamentais fica então dada por

$$
-\vec{\nabla}_{i} U=m_{i} \vec{a}_{i}
$$

com $m_{i}$ sendo o coeficiente de inércia da partícula $i$.

Este resultado é interessante o suficiente para ser enfatizado. Nós partimos da hipótese da existência de partículas fundamentais clássicas que interagem entre si de maneira instantânea. As exigências de invariância das leis físicas por rotações espaciais e translações espaçotemporais implica na existência de uma função escalar que contém a informação completa da interação do sistema. Dito de outra maneira, as forças que atuam nesse sistema são sempre conservativas. Este resultado é uma versão mais forte da terceira lei de Newton para essa classe de sistemas, visto que, como vimos, ele implica em tal lei. Contudo, como sabemos, a mecânica Newtoniana é um formalismo consistente para uma classe mais geral de sistemas que pode incluir forças não-conservativas, mas com a lei de ação e reação sendo ainda respeitada. É nosso objetivo examinar como esse cenário emerge a partir dos sistemas discutidos até aqui e que obedecem à equação (28).

\subsection{Da micro à macromecânica clássica}

Mesmo podendo ser consideradas consistentemente dentro do formalismo da mecânica Newtoniana, as forças não-conservativas que modelam certos fenômenos físicos macroscópicos são entendidas como advindas efetivamente de interações mais fundamentais conservativas. Esta postura se justifica no entendimento que temos das interações fundamentais do Modelo Padrão de partículas e campos, que num sentido mais amplo (em geral é necessário considerar outras formas de energia armazenadas nos próprios campos que promovem a interação) são interações conservativas. Portanto, a todo sistema físico supõe-se a existência de uma quantidade chamada energia que se conserva. Uma das propriedades interessantes dessa grandeza é a possibilidade de transmutar-se em diferentes formas associadas às diferentes classes de graus de liberdade do sistema. Do ponto de vista mecânico, uma parte do sistema pode transferir energia para outra parte por meio da realização de trabalho. Como numa escala macroscópica certos graus de liberdade são ignorados, pois não podem ser resolvidos por instrumentos de medida apropriados apenas para essa escala, atribuímos uma dissipação da energia mecânica ou, de forma equivalente, descrevemos os fenômenos com forças efetivas não-conservativas. Contudo, como discutimos, de fato o que está ocorrendo é um fluxo de energia para graus 
de liberdade não considerados na escala macroscópica. Provavelmente um exemplo simples do que acabamos de dizer possa ser mais elucidativo. Quando estamos tentando resolver o problema de um bloco deslizando sobre um plano inclinado, não estamos preocupados na dinâmica dos graus de liberdade microscópicos das partículas que compõem o bloco e sim no movimento médio dessas partículas que corresponde ao movimento do centro de massa do sistema. Se existe atrito entre o plano e o bloco, haverá um fluxo de energia para essas partículas microscópicas aumentando a temperatura do bloco — que pode ser estimada estatisticamente como a energia cinética média das partículas. Contudo, do ponto de vista mecânico, não estamos interessados nos fenômenos térmicos e simplesmente ignoramos essa dinâmica microscópica tratando o sistema com uma força dissipativa de atrito.

É importante frisarmos que o significado de efetivo nessa discussão é ligeiramente diferente do que foi empregado anteriormente em conexão com teorias efetivas emergentes de uma teoria mais fundamental. Naquele contexto, dizíamos que os graus de liberdade mais fundamentais eram descritos com uma certa teoria microscópica e o efeito da integração de alguns desses modos pode se manifestar como correção na dinâmica dos modos característicos de mais baixa energia, que por sua vez são descritos em termos de uma teoria distinta. Naquele sentido, não ignoramos os graus de liberdade microscópicos, mas descrevemos seus efeitos de outra maneira. No presente contexto, certos graus de liberdade do sistema são de fato ignorados.

Ilustremos essa questão com uma discussão quantitativa do desacoplamento da dinâmica entre os graus de liberdade de um sistema descrito classicamente e o efeito de ignorarmos uma parte dessa dinâmica. Consideremos a interação entre dois objetos macroscópicos $O_{1}$ e $O_{2}$ que são compostos por $N_{1}$ e $N_{2}$ partículas clássicas fundamentais, respectivamente. Supondo o sistema $\mathrm{O}_{1}+\mathrm{O}_{2}$ isolado, temos que a segunda lei de Newton 29 sobre cada uma das $N_{1}+N_{2}$ partículas é dada por

$$
\vec{F}_{i}^{\alpha}=\sum_{\beta} \sum_{j=1}^{N_{\beta}} \vec{F}_{i j}^{\alpha \beta}=m_{i}^{\alpha} \vec{a}_{i}^{\alpha}, \quad \text { com } \vec{F}_{i i}^{\alpha \alpha}=0,
$$

onde $\alpha, \beta=1,2$ rotulam os objetos $O_{1}$ e $O_{2}$ e os índices $i$ e $j$ rotulam as partículas no interior desses sistemas. Assim, $\vec{F}_{i j}^{\alpha \beta}$ representa a força que a $j$-ésima partícula do sistema $\beta$ exerce sobre a $i$-ésima partícula do sistema $\alpha$. Somando as equações 30 para todas as partículas de $\alpha$, obtemos

$$
\vec{F}_{\alpha \beta} \equiv \sum_{i=1}^{N_{\alpha}} \vec{F}_{i}^{\alpha}=M^{\alpha} \frac{d^{2} \vec{X}^{\alpha}}{d t^{2}}, \quad \alpha \neq \beta,
$$

$\operatorname{com} M^{\alpha}=\sum_{i=1}^{N_{\alpha}} m_{i}^{\alpha} \mathrm{e}$

$$
\vec{X}^{\alpha} \equiv \frac{1}{M^{\alpha}} \sum_{i=1}^{N_{\alpha}} m_{i}^{\alpha} \vec{x}_{i}^{\alpha}
$$

sendo o vetor posição do centro de massa do objeto $O_{\alpha}$. $\vec{F}_{\alpha \beta}$ é a força total que $O_{\beta}$ exerce sobre $O_{\alpha}$. De $(27)$, cada um dos $\vec{F}_{i j}^{\alpha \beta}$ deve satisfazer $\vec{F}_{i j}^{\alpha \beta}=-\vec{F}_{j i}^{\beta \alpha}$, de modo que podemos obter de forma imediata a validade da terceira lei de Newton para os pares de forças resultantes entre $O_{\alpha}$ e $O_{\beta}$ :

$$
\vec{F}_{\alpha \beta}=-\vec{F}_{\beta \alpha} .
$$

A equação 31 expressa o fato já conhecido de que a segunda lei de Newton é válida para os objetos compostos, com a força que causa a aceleração do centro de massa do objeto sendo a força externa resultante da interação com os demais objetos do sistema. Mesmo quando ignoramos a dinâmica das partículas constituintes destes objetos, o movimento macroscópico dos objetos como um todo obedece, portanto às três leis de Newton do movimento. Em particular, a validade da terceira lei para forças macroscópicas $\vec{F}_{\alpha \beta}$ equivale à conservação to momento total do sistema:

$$
\frac{d}{d t}\left(\vec{P}^{\alpha}+\vec{P}^{\beta}\right)=0
$$

Dessa maneira, quanto a propriedade 27) que as forças possuem de conservar o momento, o fato de ignorarmos os graus de liberdade internos não acarreta prejuízo à mesma propriedade para dinâmica macroscópica. Por outro lado, o caráter conservativo das forças microscópicas $\vec{F}_{i j}^{\alpha \beta}$, em geral, não sobrevive à essa supressão de graus de liberdade. Isto já pode ser observado da equação 31), pois para que $\vec{F}_{\alpha \beta}$ seja uma força conservativa no sentido macroscópico é necessário que exista uma função energia potencial $U^{M}$ que depende das coordenas macroscópicas $\vec{X}^{\alpha}$ e $\vec{X}^{\beta}$ e se tenha $\vec{F}_{\alpha \beta}=-\vec{\nabla}_{\vec{X}^{\alpha}} U^{M}$. No entanto, sabemos que existe a energia potencial do sistema $U\left(\vec{x}_{1}^{\alpha}, \ldots, \vec{x}_{N_{\alpha}}^{\alpha}, \vec{x}_{1}^{\beta}, \ldots, \vec{x}_{N_{\beta}}^{\beta}\right)$ e de 28$)$ e 30 , temos $\vec{F}_{\alpha \beta}=-\sum_{i} \vec{\nabla}_{\vec{x}_{i}^{\alpha}} U$. Denotando o vetor posição da $i$-ésima partícula de $O_{\alpha}$ em relação à posição do centro de massa deste objeto por $\vec{x}_{i}^{\prime \alpha}$, temos a relação

$$
\vec{x}_{i}^{\alpha}=\vec{X}^{\alpha}+\vec{x}_{i}^{\prime \alpha} \text {. }
$$

Se supusermos que a interação com o sistema $O_{\beta}$ ocorre sem transferência de energia para os graus de liberdade internos, podemos fazer $d \vec{x}_{i}^{\prime \alpha}=0$ e, portanto, $d \vec{x}_{i}^{\alpha}=$ $d \vec{X}^{\alpha}$, de modo que $\vec{F}_{\alpha \beta}=-\vec{\nabla}_{\vec{X}^{\alpha}} \sum_{i} U$ e obteríamos uma força conservativa. No entanto, quando há fluxo para tais graus de liberdade internos $\vec{\nabla}_{\vec{X}^{\alpha}} \neq \vec{\nabla}_{\vec{x}_{i}^{\alpha}}$ e, portanto, não teremos uma força macroscópica conservativa.

Pode também ser interessante repetir o argumento acima em termos da análise explícita do teorema trabalhoenergia. Para isso, consideramos novamente a segunda lei (30) aplicada às partículas constituintes do corpo $O_{\alpha}$. Fazendo o produto escalar desta equação pelo deslocamento infinitesimal da partícula correspondente $d \vec{x}_{i}^{\alpha} \mathrm{e}$ somando sobre todas as $N_{i}^{\alpha}$ partículas, obtemos

$$
\sum_{i} \vec{F}_{i}^{\alpha} \cdot d \vec{x}_{i}^{\alpha}=\sum_{i} m_{i}^{\alpha} \vec{a}_{i}^{\alpha} \cdot d \vec{x}_{i}^{\alpha} .
$$


Tomando a diferencial da relação (35) e usando o resultado em (36), temos

$$
\begin{aligned}
& \sum_{i} \vec{F}_{i}^{\alpha} \cdot d \vec{X}^{\alpha}+\sum_{i} \vec{F}_{i}^{\alpha} \cdot d \vec{x}_{i}^{\prime \alpha} \\
& =\sum_{i} m_{i}^{\alpha}\left(\frac{d \vec{V}^{\alpha}}{d t}+\frac{d \vec{v}_{i}^{\prime \alpha}}{d t}\right) \cdot\left(\vec{V}^{\alpha}+\vec{v}_{i}^{\prime \alpha}\right) d t
\end{aligned}
$$

De 32 e 35, mostramos que $\sum_{i} m^{i} \vec{x}_{i}^{\prime} \equiv 0$, o que também implica em $\sum_{i} m^{i} \vec{v}_{i}^{\prime}=0$. Assim, podemos reescrever a equação (37) da seguinte forma:

$$
d W^{M}+d W^{I}=d T^{M}+d T^{I},
$$

$\operatorname{com} d W^{M}=\sum_{i} \vec{F}_{i}^{\alpha} \cdot d \vec{X}^{\alpha}$ e $d W^{I}=\sum_{i} \vec{F}_{i}^{\alpha} \cdot d \vec{x}_{i}^{\prime \alpha}$ sendo o que definimos por trabalho macroscópico e interno, respectivamente. Da mesma maneira, temos as correspondentes variações das energias cinéticas $T^{M}=\frac{1}{2} M^{\alpha}\left(\vec{V}^{\alpha}\right)^{2}$ e $T^{I}=\sum_{i} \frac{1}{2} m_{i}^{\alpha}\left(\vec{v}_{i}^{\prime \alpha}\right)^{2}$. Agora, sendo forças conservativas do ponto de vista microscópico, o lado esquerdo de (38) deve corresponder à variação da energia potencial total $d U$ do sistema e $d W^{I}$ pode ser entendido como um fluxo de calor $₫ Q$ para o interior sistema. Além disso, como podemos mostrar que $d W^{I}=d T^{I}$ vale independentemente, temos

$$
d U=-d T^{M}-d Q
$$

que expressa a conservação da energia total do sistema. Ou seja, do ponto de vista macroscópico a força $\sum_{i} \vec{F}_{i}^{\alpha}$ é não-conservativa e devemos considerar geração de calor para recuperarmos a lei de conservação da energia.

Mostramos então que numa escala macroscópica podemos incluir no formalismo da mecânica, forças em geral não-conservativas. Neste caso, a mecânica clássica emergente satisfaz às três leis de Newton na forma como enunciamos na Sec. 2 .

\section{Considerações finais}

Neste trabalho, investigamos a naturalidade da mecânica clássica como sendo uma teoria efetiva advinda de uma descrição mais fundamental e quando assumimos alguns princípios gerais como homogeneidade e isotropia espacial, homogeneidade temporal e preditibilidade. O que particularmente tentamos argumentar é que as hipóteses da validade do princípio da relatividade (invariância das leis físicas por boosts Galileanos) e de o estado de uma partícula ser univocamente dado por sua posição e velocidade que, de fato são justificadas experimentalmente, podem ser obtidas de maneira natural se a mecânica clássica for pensada como uma teoria efetiva.

Para obtermos as leis de Newton da mecânica clássica conforme conhecemos, separamos a análise em termos de uma mecânica clássica efetiva e macroscópica e uma extrapolação ideal desta onde os fenômenos são descritos em termos de partículas fundamentais clássicas. Neste cenário ideal, iniciamos a investigação do movimento de uma partícula livre e da prescrição unívoca de um estado inicial. Com as hipóteses de simetria, obtemos uma expansão em termos de derivadas de ordem par da posição da partícula começando com o termo de segunda derivada e condicionamos que mecânica clássica seja entendida como uma teoria efetiva válida para descrição dos fenômenos numa escala macroscópica, $\Delta t \gg T_{0}$, com $T_{0}$ sendo a escala característica da teoria mais fundamental. Usando argumentos de análise dimensional inspirados na descrição de teorias efetivas com o grupo de renormalização de Wilson [15], estimamos a ordem de grandeza dos coeficientes das derivadas de ordem superior da expansão da lei da dinâmica das partículas livres. Supondo a escala $T_{0}$ advinda da combinação da escala de comprimento atômica e da velocidade da luz, estimamos que as correções à lei da inércia $\vec{a}=0$ são suprimidas por fatores da ordem de $10^{-30}$ para fenômenos macroscópicos, fornecendo uma robusta verificação da validade desta lei.

Com a condição de instantaneidade da interação entre as partículas fundamentais, e os mesmos critérios de simetria, demonstramos a existência de um potencial de interação entre estas partículas e a validade da terceira lei de Newton.

A introdução da classe mais geral de forças não-conservativas foi analisada em conexão com a discussão da emergência de uma mecânica clássica macroscópica a partir do cenário inicial idealizado. Como usualmente se discute as leis de Newton para um sistema de partículas, obtemos o desacoplamento da dinâmica dos graus de liberdade macroscópicos dos demais graus de liberdade microscópicos que não podem ser resolvidos com instrumentos com precisão finita. A supressão desses graus de liberdade não resolvidos nos fornece a dinâmica em termos de forças, em geral, não-conservativas, mas que de outro modo satisfaz às três leis de Newton conforme bem conhecemos.

\section{Referências}

[1] Galileo Galilei, Dialogue Concerning the Two Chief World Systems: Ptolomaic and Copernican (Random House Publishing Group, Berkeley and Los Angeles, 2001).

[2] Galileo Galilei, Dialogues Concerning Two New Sciences, (The Macmillan Comapany, New York, 1914).

[3] I. Newton, Mathematical Principles of Natural Phylosophy, (University of California Press, Berkeley, 1934).

[4] E. Noether; Mort Tavel (translator) (1971), Invariant Variation Problems, Transport Theory and Statistical Physics. 1 (3): 186-207.

[5] L. D. Landau and E. M. Lifshitz, Mechanics, (Butterworth-Heinenann, Oxford, 1976).

[6] N. A. Lemos, Mecânica Analítica, (Livraria da Física, São Paulo, 2007).

[7] A. S. Martins, Simetrias e Leis de Conservação na Mecânica Clássica, Rev. Bras. Ensino Fís., vol.21, n.1, p.33, 1999.

[8] H. M. Nussenzveig, Curso de Física Básica 1: Mecânica, (Edgard Blücher, São Paulo, 2002). 
[9] V. I. Arnold, Mathematical Methods of Classical Mechanics, (Springer-Verlag, 2nd Ed., New York, 1989).

[10] S. S. Chibeni, A Fundamentação Empírica das Leis Dinâmicas de Newton, Rev. Bras. Ensino Fís., vol.21, n.1, p.1, 1999.

[11] M. Ostrogradsky, Mem. Ac. St. Petersbourg VI 4 (1850) 385.

[12] J. Polchinski, Effective Field Theories and the Fermi Surface, TASI 1992, arXiv:9210046.

[13] P. R. S. Gomes, An Introduction to Emergent Symmetries, Int. J. Mod. Phys. A31 (2016), arXiv:1510.04492.

[14] Uma discussão didática sobre a utilidade da análise dimensional pode ser encontrada em: D. Trancanelli, Grandezas físicas e análise dimensional: da mecânica à gravidade quântica, Rev. Bras. Ensino Fís., vol.38, n.2, e2505, 2016.

[15] K. Wilson e J. Kogut, The Renormalization group and the $\epsilon$ expansion, Phys.Rept. 12 (1974) 75-200. 